\title{
INTEGRASE INHIBITORS: Why do We NeEd a NeW Drug Class For HIV Therapy?
}

\author{
Jürgen Kurt Rockstroh \\ Department of Internal Medicine I, Bonn University, Germany
}

\section{INTRODUCTION}

With the introduction of highly active antiretroviral therapy (HAART) in 1996, a dramatic decline in HIV associated morbidity and mortality has been observed, which represents one of the biggest medical achievements in the history of medicine and infectious diseases up to the very day. Despite the possibilities of long term control of HIV replication however, it became evident quite rapidly that issues around short-term and long-term toxicities, as well as adherence challenges and the emergence of mutations conferring resistance to the commonly used antiretroviral therapies limited the long-term success rates of HAART. Correspondingly, a quest has remained for the development of better tolerated and easier to take drug regimens, aiming at improving long-term outcome of HIV therapy.

Moreover, it needs to be pointed out, that in developed countries with access to ART the HIV population is aging and HIV-infected men who have sex with men (MSM) over 60 years of age have been increasing; in Australia for instance in number by $12 \%$ per year since 1995 [1]. With the increasing number of older patients and the higher frequency of co-morbidities and requirement for co-medications, issues around drug-drug-interactions and impact of respective antiretrovirals on underlying other diseases is of increasing importance. Therefore, at least from patients and physicians side, the development of new drug classes remains crucial, not only to obtain new drugs with remaining antiviral activity in the patient population which already has developed drug resistance to the commonly available drug classes, but also for improving the long-term tolerability of antiretrovirals in general. A different mechanism of action of a respective new drug class promises a potentially different side effect profile as well as depending on the respective metabolisation pathway a somewhat different potential for drug-drug interactions, thereby possibly facilitating further concomitant therapy.

In the current supplement of the "European Journal of Medical Research" we address some of the most burning questions around the use of integrase inhibitors in clinical practice and introduce not only the most recent data but also outline the possible future role of integrase inhibitors in the treatment of HIV.

\section{INTEgRASE INHIBITORS - A NeW MOdE OF ACTION}

The molecular basis of the mechanism of action of an integrase inhibitor is provided by Jean-Francois Mouscadet and Luba Tchertanov in this supplement [2]. In principle, productive infection with HIV-1 requires three key steps in the viral replication. First: reverse transcription of viral genomic RNA into viral cDNA by the viral reverse transcriptase; Secondly, integration of viral cDNA into the host cell genome using the viral integrase; and thirdly cleavage of newly synthesized viral polypeptides by the viral protease into individual viral proteins during new virion assembly. Correspondingly, multiple reverse transcriptase and protease inhibitors have been used for more than 13 years to treat HIV-infected individuals, but only recently has the viral integrase enzyme emerged as an alternative clinically validated target to block HIV-1 replication. Overall, three essential steps for the actions of HIV integrase have been identified: binding of integrase to viral DNA, formation of the pre-integrase complex, and strand transfer. The integrase inhibitors in clinical use and more advanced development such as raltegravir and elvitegravir (raltegravir has already being licensed for use in naive and experienced patients whereas elvitegravir is currently still in Phase 3 clinical development) both selectively inhibit strand transfer thereby preventing viral DNA integration into the CD4+ cell chromosome. With regard to metabolization pathways it is important to point out that raltegravir is not metabolized via the cytochrome p450 system therefore, promising less drug-drug interactions than seen within the PI and NNRTI class. Elvitegravir on the other hand is dependant on the co-formulation with a PK-booster thereby suggesting relevant interactions with other drugs metabolized by the CYP $3 \mathrm{~A} 4$ pathway. An overview of the so far known published drug-drug interactions for raltegravir is provided by David Burger in this supplement [3].

\section{INTEGRASE INHIBITORS - FOR USE IN TrEatMent NAIVE PATIENTS}

Various highly efficacious first-line treatment options exist in the currently available HIV treatment guide- 
lines. In clinical practice more than $50 \%$ of patients are started on a TDF/FTC/efavirenz based regimen which is available in a fix-dose combination as one pill per day. And although this truly simple, highly efficacious treatment has improved long-term treatment outcome in HIV-infected individuals tremendously, concerns remain with regard to short and long-term CNS toxicity typically observed under efavirenz therapy. Moreover, primary NNRTI resistance, teratogenicity issues with efavirenz in women of child-bearing potential, lipid changes, drug-drug interactions, as well as the low genetic barrier and risk for resistance emergence in patients with adherence problems highlight the challenges with the current gold standard of antiretroviral therapy. In the pivotal first-line antiretroviral treatment study, comparing antiretroviral efficacy and safety profile of efavirenz/TDF/FTC with raltegravir/TDF/FTC, comparable impressive virological success rates were found for both regimens $[4,5]$. Due to the differences in chemical structure and mode of action however, less CNS toxicity as well as a distinct lipid profile for the raltegravir treated patients was found, thereby making the integrase inhibitor an attractive alternative in patients not tolerating efavirenz. In this supplement Lazzarin and colleagues summarize the findings of the various trials with integrase inhibitors in the antiretroviral treatment-naïve population and try to outline the future use of this class in the naive patient population [6].

\section{UsE OF INTEGRASE INHIBITORS IN TREATMENT EXPERIENCED PATIENTS}

Although the number of virological failures in all HIV patient cohorts worldwide under modern potent HAART is declining significantly, there is a group of patients who started HIV therapy when AZT monotherapy became available in 1987 and have subsequently frequently received functional mono-therapies of new drugs as they came into the market in combination with a backbone of already used and mostly resistant other antiretroviral drugs with only little if any retained antiviral activity. Therefore, the introduction of integrase inhibitors as a new drug class was awaited with great excitement as it offered the possibility of composing a fully active new antiretroviral regimen, particularly as very potent $2^{\text {nd }}$ generation protease inhibitors as well as $2^{\text {nd }}$ generation NNRTIs became available around the same time, promising retained antiviral activity even if some resistance conferring mutations from that class may have occurred previously. Indeed, in the treatment experienced trials with the integrase inhibitor raltegravir (BENCHMRK) unexpectedly high treatment success rates were obtained in a previously triple class drug experienced patient population with multiple mutations in all 3 classes $[7 \mid$. This was particularly true when raltegravir was combined with other active drugs such as darunavir or enfurvitide. More recently, results from the French TRIO trial were presented where, again in a highly pretreated 3-drug class experienced patient population, therapy with raltegravir/etravirine and darunavir led to over $90 \%$ of patients being undetectable after 24 weeks of therapy [8]. These findings clearly high- light the new treatment possibilities in treatment experienced patients in 2009 . The results from the various trials in this often challenging to treat patient population are discussed in this issue by Jose Gatell [9].

\section{TREATMENT OF INTEGRASE INHIBITORS IN Special Patient Populations}

The impressive antiviral potency, the excellent tolerability profile and the lack of significant drug-drug interactions make raltegravir an attractive option for many patients with HIV infection. It may play an important role in patients undergoing solid organ and bone marrow transplantation, patients receiving chemotherapy for malignant disease. It may also be a promising option in patients with hepatitis or $\mathrm{HIV} / \mathrm{TB}$ co-infection. In this supplement the possible role of integrase inhibitors in HIV therapy for hepatitis coinfected patients is discussed by Martin Vogel and Mark Nelson [10].

Other groups of patients with HIV infection may also benefit from raltegravir. This will include patients with dyslipidaemia and patients who currently have side-effects from antiretroviral therapy in particular the boosted PI's. Moreover, integrase inhibitors may also play a role in pregnancy. The use of raltegravir in these special patient populations will be discussed in more detail by Margret Johnsen [11].

\section{RESISTANCE DEVELOPMENT UNDER INTEGRASE INHIBITORS}

The last review in this supplement eventually deals with the issues around integrase resistance development [12]. Resistance to raltegravir can require only a single point mutation. Indeed in the BENCHMRK trial among the subjects in the raltegravir group with virologic failure who underwent genotypic resistance testing, approximately two thirds showed at least one of the known resistance mutations to raltegravir by week 48. As expected, the prevalence of resistance mutations was lower among isolates from patients who were given (in addition to raltegravir) two or more active drugs (33\%), as compared with none $(78 \%)$. These findings highlight once again the importance of combining at least 2, preferably 3, active drugs into a new antiretroviral combination therapy rather than administering functional mono-therapies with only one active drug. Clearly resistance emergence is to be avoided under all circumstances as cross-resistance limits the further options in the integrase drug class. Resistance studies have described Q148R to be the prime mutation conferring resistance to both raltegravir and elvitegravir. More recently, other new compounds such as S/GSK1349572 have been presented which demonstrated activity in vitro against laboratory HIV strains that contain mutations associated with raltegravir and elvitegravir resistance (e.g., Q148R, N155H and Q148H/G140S). These data suggest that S/GSK1349572 has the potential to be used after a previous first generation integrase inhibitor failure. However, the clinical significance of resistance data continues to be evaluated, and these observations need to be confirmed in clinical studies. 


\section{SUMMARY}

The introduction of integrase inhibitors as a new drug class for treatment of HIV have added a very potent and extremely well tolerated new treatment option to the HIV-armentarium. Particularly in patients with previously developed drug resistance, the availability of new active drugs offers great hope. Moreover, the in general excellent safety profile offers numerous opportunities for all lines of therapy and special patient populations. Clearly more data is needed to further explore and define the future role of integrase inhibitors in HIV-care.

\section{REFERENCES}

1. Murray JM, McDonald AM, Law MG. Rapidly ageing HIV epidemic among men who have sex with men in Australia. Sex Health. 2009;6: 83-86.

2. Mouscadet JF, Tchertanov L. Raltegravir: molecular basis of its mechanism of action. Eur J Med Res 2009; 14 (Suppl III): $5-16$

3. Burger D. Drug-drug interactions with raltegravir. Eur J Med Res 2009;14 (Suppl III): 17-21

4. Markowitz M, Nguyen BY, Gotuzzo E et al. Rapid and durable antiretroviral effect of the HIV-1 integrase inhibitor raltegravir as part of combination therapy in treatment-naive patients with HIV-1 infection: results of a 48week controlled study. J Acquir Immune Defic Syndr 2007;46: 125

5. Markowitz M, Nguyen BY, Gotuzzo E et al. Sustained antiretroviral effect of raltegravir after 96 weeks of combination therapy in treatment-naïve patients with HIV-1 infection. J Acquir Immune Defic Syndr 2009; 52: 350-356
6. Cossarini F, Castagna A, Lazzarin A. Raltegravir in treatment-naive patients. Eur J Med Res 2009;14 (Suppl III): 22-29

7. Steigbigel RT, Cooper DA, Kumar PN, et al. BENCHMRK Study Teams. Raltegravir with optimized background therapy for resistant HIV-1 infection. N Engl J Med. 2008;359: 339-354

8. TRIO Yazdanpanah Y, Fagard C, Descamps D et al., ANRS 139 TRIO Trial group High rate of virologic suppression with raltegravir plus etravirine and darunavir/ ritonavir among treatment-experienced patients infected withmultidrug-resistant HIV: results of the ANRS 139 TRIO trial. Clin Infect Dis. 2009;49: 1441-9.

9. Gatell J. The use of integrase inhibitors in treatment-experienced patients. Eur J Med Res 2009;14 (Suppl III): 30-35

10. Vogel M,, Nelson M: HIV/HCV coinfection: Which role can new antiretrovirals such as integrase inhibitors play? Eur J Med Res 2009;14 (Suppl III): 36-42

11. Johnson M: Raltegravir use in special populations Eur J Med Res 2009;14 (Suppl III): 43-46

12. Clavel F: HIV Resistance to Raltegravir. Eur J Med Res 2009;14 (Suppl III): 47-54

Address for correspondencer:

Prof. Dr. med. Jürgen Rockstroh

Bonn University

Department of Medicine I

Sigmund-Freud-Str. 25

53105 Bonn, Germany

Tel.: $\quad+49 / 228 / 28716558$

Fax: + 49/228/28715034

E-mail: juergen.rockstroh@ukb.uni-bonn.de 